\title{
Estilos de cuidado materno em primatas: considerações a partir de uma espécie do Novo Mundo'
}

\author{
Michele P. Verderane* (1) \\ Patrícia Izar $\mathbb{1}$
}

Universidade de São Paulo, Instituto de Psicologia. São Paulo, SP, Brasil

\begin{abstract}
Resumo: Quando buscamos entender o comportamento humano, comparações com primatas não humanos são especialmente relevantes para identificar homoplasias (características semelhantes que evoluem independentemente em diferentes espécies). Neste artigo, apresentamos um estudo longitudinal de dois anos sobre o comportamento materno de macacos-prego (Sapajus spp.) em condições naturalísticas. Nossos resultados permitiram identificar estilos de cuidado distintos dentro de um contínuo de permissividade a proteção. 0 desenvolvimento observado do vínculo entre mães e filhotes sugere que o período de dependência de filhotes de macaco-prego envolve, além de processos de maturação física, o estabelecimento e desenvolvimento de processos psicológicos associados ao sistema de apego. É possível que a variabilidade de estilos maternos resultante da combinação de características de mães, filhotes e contextos socioecológicos, aliada ao prolongamento do vínculo de apego, pavimente caminhos para diferentes trajetórias de desenvolvimento. Como em humanos, esse pode ser um dos mecanismos pelos quais surgem e se consolidam as diferenças interindividuais nas populações adultas.
\end{abstract}

Palavras-chave: apego, interação mãe-filhote, cuidado parental, infância, Sapajus.

\section{Introdução}

A ideia de que as mulheres possuem uma predisposição natural incondicional para apegar-se e cuidar de um bebê, o que chamamos de instinto materno, é colocada em cheque pelos casos de abandono e até mesmo neonaticídio de bebês por suas mães, cuja ocorrência tem sido registrada ao longo da história e em diferentes culturas (Badinter, 1985). Para Sarah Hrdy (1999), esses casos extremos revelam que a predisposição feminina para o cuidado materno varia num contínuo de acordo com as contingências que afetam sua subsistência, as necessidades de seus filhos e seu próprio futuro reprodutivo. Na nossa vida moderna, além de condições econômicas e de apoio social, essas contingências incluem expectativas culturais, papéis sexuais, honra e vergonha, preferências sexuais e expectativa do futuro. Hrdy desenvolveu sua tese a partir da perspectiva etológica, caracterizada pela análise comparativa de animais humanos e não humanos, a fim de compreender os estímulos eliciadores e mecanismos internos reguladores de um comportamento, como esse comportamento se modifica ao longo do desenvolvimento, qual a história evolutiva desse comportamento e qual seu valor de sobrevivência (Tinbergen, 1963). A perspectiva comparativa permite identificar características presentes numa espécie que são partilhadas por espécies próximas,

Endereço para correspondência: miverderane@gmail.com

1 Agradecemos à Liliane Milanelo, por autorizar a realização dessa pesquisa no Parque Ecológico do Tietê (PET), e à Fapesp, pelo apoio financeiro (03/03095-0). sugerindo uma ancestralidade comum (homologia), ou que são exclusivas. Quando se identificam homoplasias, isto é, quando as mesmas características (fisiológicas, morfológicas, psicológicas) evoluem independentemente em diferentes espécies, sob as mesmas pressões seletivas e servem à mesma função, podemos inferir adaptação.

Devido às diferenças sexuais na fisiologia reprodutiva (produção de gametas, fertilização e gestação interna), em geral, fêmeas são mais predispostas do que machos a investirem em cuidado parental. Assim, nesse artigo trataremos exclusivamente do cuidado parental materno. Pela perspectiva etológica, o cuidado materno evoluiu por aumentar as chances de sobrevivência dos filhotes, mas às custas da diminuição da habilidade das mães em investir em proles futuras (Trivers, 1972). Do ponto de vista da aptidão (sucesso reprodutivo) individual, essa condição cria um "conflito evolutivo". Para as mães, é mais vantajoso encerrar os cuidados aos seus filhotes atuais tão logo eles sejam capazes de sobreviver por conta própria. Para os filhotes, é mais vantajoso receber cuidados pelo maior tempo possível. À luz da teoria da Evolução, espera-se que quando os custos do cuidado à prole excedem os seus benefícios, a seleção favoreça o comportamento de rejeição materna (Trivers, 1974). A vantagem para as mães em recusar o cuidado dependerá das habilidades de seus filhotes para sobreviverem independentemente.

Para um cuidado materno efetivo é fundamental que haja proximidade física entre mães e filhotes. A Teoria do Apego (Ainsworth, 1979; Bowlby, 1969) propõe que o vínculo mãe-filhote é uma característica 
adaptativa de muitas espécies animais, incluindo a humana, moldada pela seleção natural por promover a proteção do imaturo. O sistema de apego consiste em um conjunto de comportamentos que promovem a formação e manutenção do vínculo de proximidade e a interação socioafetiva entre mãe e filhote. Esse sistema é essencial para o desenvolvimento da criança e forma a base para as futuras relações sociais e desenvolvimento da personalidade. Sua função primária é garantir a proximidade entre o filhote e seu cuidador primário (a mãe, no caso dos mamíferos), assegurando a proteção e o conforto essenciais para o seu desenvolvimento físico (Ainsworth \& Bowlby, 1991).

Nesse cenário, a transição entre a dependência de cuidados maternos para a sobrevivência e a vida autossuficiente é muitas vezes marcada por conflitos entre mães e filhotes (Altmman, 1980; Trivers, 1974). Em primatas, a infância é definida como o período em que o jovem é fisicamente dependente de sua mãe para a sobrevivência e a idade do desmame alimentar e locomotor (i.e., filhote deixa de se alimentar do leite materno e de ser transportado pela mãe) é o critério comumente utilizado para determinar a duração da infância (Lonsdorf, 2017; Pereira \& Altmann, 1985). Contudo, o momento e a rigidez com que uma mãe rejeita seu filhote pode ser bastante variável, dentro de limites de flexibilidade comportamental de cada espécie, sugerindo a participação de outros processos na equação dessa dinâmica (Verderane, 2005). De acordo com a Etologia, a expressão do comportamento materno é o resultado final da combinação entre o repertório da espécie e características individuais de mães e filhotes e do entorno socioecológico em que eles vivem (Altmman, 1980).

Os primatas não humanos são especialmente atraentes para estudos comparativos da relação mãefilhote na perspectiva da Etologia. Por um lado, habitando uma grande variedade de ambientes, permitem investigar hipóteses sobre causas de flexibilidade comportamental. Por outro, pela ancestralidade comum mais próxima e por reunir diversas características homólogas com humanos, oferecem uma janela para a investigação das bases evolutivas do comportamento humano.

Com exceção de algumas espécies com cuidado biparental, particularmente comum na família Callitrichidae (Yamamoto et al., 2014), o cuidado parental materno é a regra entre as mais de 300 espécies de primatas não humanos (Maestripieri, 2001), sendo as mães as principais responsáveis por alimentar, transportar, proteger e inserir o filhote no ambiente físico e social (Berman, 1980; Verderane, 2005). Portanto, durante a infância, que é particularmente longa na ordem primata, a mãe representa o vínculo social mais forte do filhote. Esse vínculo prolongado com a mãe, além de fundamental ao cuidado materno efetivo, também pode contribuir para a aprendizagem dos filhotes. Por exemplo, a mãe pode servir de modelo para aquisição de técnicas de forrageamento (Maestripieri, Ross, \& Megna, 2002), ou como base segura em situações em que o filhote se depara com novos desafios (Okamoto-Barth, Tanaka, Kawai, \& Tomonaga, 2007). A forma como as mães cuidam das necessidades de seus filhotes, contudo, é variável e são essas variações que definem o que chamamos de estilo de cuidado materno.

De acordo com a literatura, exclusivamente baseada em estudos de espécies do Velho Mundo ${ }^{2}$, o estilo de cuidado materno emerge da combinação de comportamentos de rejeição e de proteção que as mães direcionam aos seus filhotes (Altmann, 1980). Existem dois modelos que visam classificar os estilos maternos de primatas não humanos. De acordo com Altmann, no modelo mais clássico, as mães são classificadas como restritivas (ou protetoras) ou permissivas (ou laissezfaire). Mães protetoras amamentam, transportam, catam e mantêm maior proximidade física com seus filhotes; enquanto mães rejeitadoras exibem perfil oposto (Altmann, 1980). Já Maestripieri (1999) defende que a combinação dos dois componentes do cuidado materno, a proteção e a rejeição, geram quatro estilos de cuidado possíveis; (1) protetor: muita proteção e pouca rejeição; (2) controlador: muita proteção e rejeição; (3) rejeitador: pouca proteção e muita rejeição; (4) permissiva; pouca proteção e rejeição.

Como ocorre em humanos (Carvalho, Seidlde-Moura, Martins, \& Vieira, 2014), as diferenças no estilo de cuidado materno de primatas não humanos, seja entre populações de mesma espécie ou entre indivíduos de uma mesma população ou grupo social, decorrem de variações em características individuais de mães (como a experiência reprodutiva, o posto na hierarquia de dominância e o temperamento) e filhotes (como o sexo e o temperamento) e da interação com o entorno socioecológico no qual a díade vive (como riscos de morte para mães e filhotes, padrão de oferta de alimento, tamanho e composição do grupo social) (Dettmer et al., 2016; Fairbanks, 2003; Li, Ren, Li, Zhu, \& Li, 2013; Saltzman, \& Maestripieri, 2011). Por exemplo, mães que percebem mais riscos aos seus filhotes, tais como fêmeas subordinadas e primíparas, e aquelas em sociedades despóticas, de maneira geral exibem um estilo mais protetor do que aquelas que vivem em contextos menos arriscados (Li et al., 2013; Verderane \& Izar, 2014). Em macacos rhesus (Macaca mulatta), mães que criam seus filhotes na presença da família têm estilo de cuidado permissivo, enquanto mães com pouco ou nenhum parente no grupo social tendem a ser restritivas com seus filhotes (Berman, Rasmussen, \& Stephen, 1997). Para diversas espécies de cercopitecoides (grupo de primatas do Velho Mundo), mães que vivem em ambientes imprevisíveis são menos responsivas às necessidades de seus filhotes, especialmente se as demandas para sobrevivência

2 Os primatas do Velho Mundo pertencem à subordem Catarrhini, que divergiram há pelo menos 40 milhões de anos das espécies do Novo Mundo, pertencentes à subordem Platyrrhini (Chatterjee, Ho, Barnes, \& Groves, 2009). 
forem elevadas e o suprimento alimentar imprevisível (Rosenblum \& Andrews, 1994).

Paradoxalmente, apesar do grande avanço das últimas décadas na compreensão do estilo de cuidado materno e das variáveis que o afetam, observa-se, ainda, uma lacuna na literatura, que se baseia em um número restrito de espécies do Velho Mundo, sendo a maioria dos estudos referente a grupos e populações cativos. Quando buscamos entender o comportamento humano, a comparação com primatas do Novo Mundo é especialmente relevante para identificar homoplasias. Assim, neste artigo apresentamos resultados de um estudo de dois anos sobre o comportamento materno de macacos-prego (gênero Sapajus) em condições naturalísticas, que permitiram identificar estilos de cuidado distintos dentro de um contínuo de permissividade a proteção e o padrão de desenvolvimento do vínculo de apego entre mães e filhotes. O macaco-prego é um primata Neotropical que exibe grande flexibilidade comportamental em função de variabilidade de ambientes ocupados, com ampla distribuição geográfica, e inúmeras convergências adaptativas com os pongídeos ${ }^{3} \mathrm{e}$ humanos, incluindo imaturidade do filhote ao nascimento e prolongamento da infância (Fragaszy, Visalberghi, \& Fedigan, 2004). Essas características fazem dos macacosprego modelos ideais para a investigação ora proposta, considerando que estudos com espécies filogeneticamente distantes das do Velho Mundo e dos humanos podem fornecer evidências sobre nossas adaptações psicológicas e comportamentais, destacando homologias com o padrão de cuidado materno e apego mãe-bebê.

\section{Métodos}

\section{Local e sujeitos do estudo}

Nosso estudo foi realizado no Parque Ecológico do Tietê (PET), na cidade de São Paulo, com um grupo de macacos-prego que habita uma área reflorestada de 19,5ha dentro dos limites do PET. O local abriga uma variedade de espécies botânicas e animais, muitas das quais são consumidas pelos macacos-prego, mas sem a presença de predadores naturais de primatas. Os macacos locomovem-se livremente nessa área e alimentam-se de recursos naturalmente disponíveis na mata e de uma mistura de frutas e verduras fornecida diariamente pelos biólogos do PET (Verderane, 2005).

Os sujeitos desse estudo foram cinco díades mãefilhote (1 filhote macho e 4 fêmeas de 3 mães multíparas e 2 primíparas ${ }^{4}$ ) de um grupo de macacos-prego composto por 22 indivíduos ( 5 machos e 5 fêmeas adultos, 3 machos e 2 fêmeas juvenis e 2 machos e 5 fêmeas infantes Tabela 1) numa organização similar àquela de grupos de macacos-prego selvagens (Izar \& Ferreira, 2007). Nossos

3 Família de primatas com ancestralidade comum mais próxima à dos humanos, inclui o orangotango, o gorila e o chimpanzé.

4 O termo primípara refere-se à fêmea que têm filhote pela primeira vez, e multípara refere-se à fêmea que já teve mais de um filhote. dados constituem observações naturalísticas dessas cinco díades ao logo do período completo entre o nascimento e o $15^{\circ}$ mês de vida dos filhotes. Usamos dados adicionais e análises de medidas repetidas para investigar se as três mães multíparas do nosso estudo foram consistentes na forma como amamentaram e transportaram sucessivos filhotes. Essas análises foram realizadas com oito filhotes - cinco do presente estudo e três de um estudo anterior com a mesma população (Resende, 2004) -, totalizando quatro filhotes da Física (Fractal, Filó, Frida e Fábio), dois da Ana (Ada e Angélica) e dois da Janete (Janeiro e Jujuba).

\section{Método de amostragem e variáveis comportamentais}

Os dados foram coletados ao longo de 26 meses, entre fevereiro de 2002 e abril de 2004, totalizando 422 horas de observação de mães e filhotes. Os registros comportamentais foram feitos por M.P.V., através do método Animal Focal (Altmann, 1974), com intervalo amostral de 10 minutos e registros instantâneos a cada minuto. O primeiro mês de estudo foi dedicado ao treinamento da pesquisadora com o método de amostragem, as categorias comportamentais e a calibragem para aprender a estimar as distâncias entre mães e filhotes. Cada mãe e cada filhote foram observados pelo menos uma vez por semana, segundo ordem previamente determinada por sorteio, respeitando-se distribuição equitativa entre manhã (06:0012:00h) e tarde (12:01-17:00h), intervalo mínimo entre as observações de um mesmo indivíduo de uma hora e não mais do que duas observações de um mesmo indivíduo em cada período de um mesmo dia.

Para investigar o estilo de cuidado materno, utilizamos as seguintes variáveis comportamentais (frequência normalizada por tempo de observação da díade): (1) Amamentação; (2) Transporte materno; (3) Catação (i.e., mãe higieniza o filhote); (4) Partilha de alimentos (i.e., mãe compartilha alimento sólido com o filhote); (5) Rejeição (i.e., bater ou ameaçar o filhote); (6) Manutenção da proximidade com o filhote (ver adiante).

Para investigar o processo de independência do filhote, utilizamos as variáveis comportamentais de alimentação e locomoção independentes (frequência normalizada por tempo de observação do filhote). Os últimos registros de amamentação e transporte materno de cada filhote foram utilizados para determinar as idades de desmame alimentar e locomotor, respectivamente.

$\mathrm{O}$ desenvolvimento do vínculo de proximidade física entre mãe e filhote foi avaliado com base em dois critérios. Para determinar qual membro da díade foi mais responsável por manter e restaurar a proximidade física entre a díade, utilizamos o índice de proximidade (Hinde \& Atkinson, 1970). Para avaliar a dinâmica da associação espacial, calculamos o tempo (proporção de amostradas focais) em que mãe e filhote permaneceram às distâncias de: (1) em contato até 1 metro; (2) 1,1 metros até 10 metros; (3) a mais de 10 metros um do outro. 


\section{Resultados e discussão}

\section{Estilos de cuidado materno de macacos-prego}

Como dissemos anteriormente, o estilo de cuidado materno em primatas não humanos consiste em diferenças individuais em parâmetros de cuidado maternal persistentes ao longo da criação de sucessivos filhotes. Dessa forma, primeiramente verificamos se as mães de macacos-prego do nosso estudo apresentaram estilos de cuidado distintos. Utilizamos a abordagem tradicional de contínuo de proteção e rejeição materna, comparando por meio de análise de agrupamento a frequência relativa (i.e., normalizada por tempo de observação da díade) de seis comportamentos maternos: amamentação (mãe nutre o filhote através do aleitamento); transporte (mãe leva filhote junto a seu corpo); manutenção da proximidade (mãe se aproxima ou faz contato com o filhote); catação (mãe higieniza pelagem do filhote); partilha de alimento sólido (mãe permite que filhote coma do mesmo alimento que ela consome); rejeição (mãe ameaça ou bate no filhote ou impede que ele tenha acesso aos cuidados anteriormente descritos). As mães foram agrupadas de acordo com perfis semelhantes em cada uma dessas variáveis e então verificamos se os agrupamentos diferiram significativamente quanto às variáveis de cuidado.

\section{Existe variação em estilos de cuidado materno entre as fêmeas de macacos-prego estudadas?}

Identificamos três estilos de cuidado materno distintos entre as cinco fêmeas monitoradas. $\mathrm{O}$ estilo de cuidado de Ana foi caracterizado por maior frequência de amamentação que o de Física, Janete e Cisca, maior frequência de transporte que o de Física e Janete e maior responsabilidade pela manutenção da proximidade física com o filhote que todas as outras fêmeas. Os filhotes de Ana também foram desmamados mais velhos do que os filhotes de Física, Janete e Cisca. Já Física, além destas diferenças em relação a Ana, também apresentou menor frequência de amamentação e transporte e estabeleceu a independência dos filhotes mais cedo que Vavá. Física também foi a mãe que mais catou seu filhote, seguida por Janete, Ana e Vavá e Cisca, que apresentaram as taxas mais baixas. Por outro lado, Cisca foi a mãe com as maiores taxas de rejeição ao filhote, seguida por Janete, Ana, Vavá e Física (Tabela 1).

Verificamos que o estilo de cuidado das fêmeas deste estudo variou num contínuo de investimento em amamentação, transporte e manutenção da proximidade física com o filhote ao longo do seu desenvolvimento. Utilizamos a classificação de Altmann (1980), não dentro da categorização dicotômica proposta pela autora, mas dentro de um contínuo entre proteção e permissividade das mães, em que os extremos correspondem aos estilos permissivo e protetor (Figura 1). Adotamos essa nomenclatura por considerar que reflete melhor a natureza do repertório de cuidado exibido pelas fêmeas de macacos-prego. Já os componentes de catação, agonismo e partilha de alimentos com o filhote também compõem o estilo materno; mas, ou não dizem respeito à permissividade $\mathrm{e}$ à proteção, ou refletem características de temperamento da mãe, que se combinam de forma mais flexível com os demais elementos do cuidado.

Tabela 1. Tempo médio (\% do tempo de observação da díade) dedicado pelas mães de macacos-prego aos comportamentos de cuidado entre o nascimento e o $15^{\circ}$ mês de vida dos filhotes

\begin{tabular}{|c|c|c|c|c|c|c|c|c|c|}
\hline Díade & $\begin{array}{c}\text { Amamentação } \\
(\%)\end{array}$ & $\begin{array}{c}\text { Transporte } \\
(\%)\end{array}$ & $\begin{array}{l}\text { Proximidade } \\
\qquad(I P)^{a}\end{array}$ & $\begin{array}{c}\text { Catação } \\
(\%)\end{array}$ & $\begin{array}{c}\text { Partilha de } \\
\text { alimento } \\
(\%)\end{array}$ & $\begin{array}{c}\text { Rejeição } \\
(\%)\end{array}$ & $\begin{array}{c}\text { Desmame } \\
\text { alimentar } \\
(m e \hat{s})^{*}\end{array}$ & $\begin{array}{c}\text { Desmame } \\
\text { locomotor } \\
(\text { mês })^{* *}\end{array}$ & $\begin{array}{c}\text { Estilo } \\
\text { materno }\end{array}$ \\
\hline Ana/Angélica & 7,6 & 43 & -36 & 0,32 & 0,04 & 0,009 & 20 & 13 & Protetora \\
\hline Cisca/Cláudia & 6,0 & 34,5 & 17 & 0,17 & 0,04 & 0,03 & 14 & 14 & $\begin{array}{l}\text { Permissivo- } \\
\text { Intermediário }\end{array}$ \\
\hline Física/Frida & 3,8 & 27,5 & 24 & 0,14 & 0,03 & 0,003 & 10 & 8 & Permissivo \\
\hline Janete/Jujuba & 5,2 & 28 & 20 & 0,09 & 0,05 & 0,01 & $*$ & $*$ & $\begin{array}{l}\text { Permissivo- } \\
\text { Intermediário }\end{array}$ \\
\hline Vavá/Vítor & 7,7 & 41,5 & 22 & 0,2 & 0,12 & 0,008 & 18 & 16 & $\begin{array}{l}\text { Protetora- } \\
\text { intermediária }\end{array}$ \\
\hline
\end{tabular}

${ }^{a}$ Valor médio de Índice de Proximidade; * Jujuba morreu no $15^{\circ}$ mês de vida e quando ainda estava sendo amamentado e transportado pela mãe.

*Idade do filhote no último mês em que foi observado mamando.

**Idade do filhote no último mês em que foi observado sendo transportado pela mãe. 
Transporte, amamentação, manutençnao da proximidade com o infante.

\begin{tabular}{|c|c|c|c|c|}
\hline Ana & Vavá & Cisca & Janete & Física \\
\hline Estil & & & \multicolumn{2}{|c|}{ Estilo Permissive } \\
\hline
\end{tabular}

Figura 1. Classificação do estilo de cuidado materno das cinco fêmeas do estudo dentro de um contínuo de proteção e permissividade, de acordo com o investimento em amamentação, transporte e manutenção da proximidade com o filhote

Assim, Ana e Física apresentaram os dois estilos mais distintos entre si, e Janete, Cisca e Vavá apresentaram um estilo intermediário a esses dois, ou seja, com parâmetros de cuidado relacionados tanto ao estilo protetor quanto ao rejeitador. De acordo com o modelo de classificação dicotômico de Altmann (1980), Física, Janete e Cisca teriam um estilo de cuidado que obedece ao padrão permissivo, enquanto Ana e Vavá teriam um estilo de cuidado que obedece ao padrão restritivo. Já pela classificação proposta por Maestripieri (1999), Física, Janete e Cisca ainda seriam consideradas pouco protetoras, bem como Ana e Vavá muito protetoras, considerando seus investimentos em amamentação, transporte e manutenção da proximidade física com seus filhotes. Porém, devido às taxas de rejeição que direcionaram aos seus filhotes, Física seria considerada uma mãe relaxada (pouco protetora e rejeitadora ou laissez-faire), Janete e Cisca seriam consideradas mães rejeitadoras (pouco protetoras e muito rejeitadoras), Ana teria um estilo de cuidado controlador (muita proteção e rejeição) e Vavá teria um estilo de cuidado protetor (muita proteção e pouca rejeição).

À primeira vista, esses resultados enquadram-se nos perfis de estilos maternos propostos pelos modelos de Altmann (1980) e por Maestripieri (1999). Não obstante, ao inserirmos o padrão de catação e de partilha de alimento na classificação, verificamos incompatibilidades em relação às variáveis de cuidado já examinadas e às expectativas dos modelos. Por ambos, esperar-se-ia que a catação e a partilha de alimento materna fossem positivamente correlacionadas às demais variáveis de proteção, mas o que observamos foram combinações que diferiram dessas expectativas.

Sugerimos duas explicações possíveis e não excludentes. As diversas combinações entre catação, partilha de alimento e demais variáveis de cuidado materno podem indicar que esses elementos compõem o estilo materno, mas não pertencem às dimensões da proteção e rejeição. De fato, estudos mais recentes propõem a existência de uma terceira dimensão do cuidado em primatas não humanos, chamada de afetuosidade materna (Maestripieri, Hoffman, Anderson, Carter, \& Higley, 2009), que diz respeito às interações íntimas com o filhote, mas ainda pouco compreendida. Ao mesmo tempo, é possível que em macacos-prego (gênero Sapajus) - e talvez em outras espécies de primatas do
Novo Mundo - estas duas variáveis de cuidado materno combinem-se com os demais componentes do cuidado de forma mais flexível, ou até mesmo distinta, do que em primatas do Velho Mundo, que são as espécies que embasaram os sistemas de classificação por nós utilizados. Efetivamente, algumas categorias de proteção e de rejeição maternas observadas neste estudo foram bastante distintas daquelas frequentemente relatadas para primatas do Velho Mundo. Por exemplo, restringir o movimento do filhote, impedindo-o de quebrar contato ou afastar-se, ou punir suas tentativas de obter cuidado com agressão física foram comportamentos ausentes entre as mães de macacos-prego do PET. Além disso, raros foram os episódios de "recuperar filhote", em que a mãe para o que está fazendo para buscá-lo e interromper a atividade por ele realizada, ou de interromper interações sociais dos filhotes com outros membros do seu grupo social.

Esses resultados evidenciam diferenças marcantes no repertório materno entre esses dois ramos da ordem dos primatas, o que pode ser o resultado combinado de adaptações a diferentes pressões evolutivas e respostas flexíveis às características do contexto socioecológico em que mães e filhotes vivem. A estrutura social típica de macacos-prego (gênero Sapajus) oferece menor risco aos infantes comparado aos primatas do Velho Mundo (relações de dominância menos despóticas e mais igualitárias, risco de infanticídio mais baixo, maior tolerância entre fêmeas e interesse e participação de vários membros do grupo no cuidado ao filhote (Fragaszy et al., 2004; Izar et al., 2006; Izar et al., 2012; Verderane, 2005; Verderane \& Izar, 2007; Verderane, Izar, Visalberghi, \& Fragaszy, 2013). Além disso, no PET a oferta de alimento é farta e não há predadores naturais de macacos-prego e nem grandes distâncias a serem percorridas pelos indivíduos, o que torna a vida dos filhotes pouco arriscada. Essa combinação de características pode ser responsável pelas diferenças no repertório materno acima relatadas.

\section{Por que as fêmeas de macacos-prego diferem em estilos de cuidado?}

Assim como em humanos, o estilo materno em primatas não humanos pode variar conforme o entorno socioecológico em que mães e filhotes estão inseridos, como a oferta de alimento e o risco de predação, e em 
função de características intrínsecas de ambos, como o status social da mãe, o tamanho da família e o sexo do filhote (Bercovitch, 2002; Fairbanks, 2003; Li et al., 2013; Maestripieri, 1999). Em nossa pesquisa, investigamos os efeitos da paridade (i.e., experiência reprodutiva), do posto de dominância (i.e., posição individual na hierarquia de dominância do grupo) e da rede familiar e afiliativa das mães sobre seus estilos de cuidado.

As três fêmeas multíparas (Física, Ana e Janete) foram consistentes no padrão de transporte e amamentação de sucessivos filhotes, ainda que todas elas tenham mudado de posição na hierarquia de dominância do grupo (Falótico, Verderane, Resende, Ottoni, \& Izar, 2003) e vivenciado mudanças no tamanho das suas famílias, devido a mortes, nascimentos e migrações, mais de uma vez ao longo deste estudo. Além disso, Física e Ana, as duas fêmeas mais velhas do grupo, apresentaram os estilos de cuidado mais estáveis e distintos entre si. Por outro lado, as duas fêmeas primíparas (Vavá e Cisca) foram as mães menos consistentes, classificadas como protetora intermediária e rejeitadora intermediária, respectivamente. Em um trabalho subsequente com esse mesmo grupo de estudo, Carminatti (2009) observou que fêmeas primíparas $(\mathrm{N}=4)$ investiram mais em amamentação, transporte e manutenção da proximidade com seus filhotes do que fêmeas multíparas, porém as diferenças não foram significativas devido à grande variabilidade comportamental das mães primíparas.

Dessa forma, sugerimos que em macacos-prego a experiência materna afeta a estabilidade do estilo de cuidado ao filhote, com fêmeas multíparas apresentando perfis consistentes ao longo do tempo e pouco permeável às flutuações sociais. Não obstante, diferente do que se observa em espécies do Velho Mundo, a paridade não determinou o estilo adotado por cada fêmea.

\section{Vínculo mãe-filhote em macacos-prego: apego e conflito}

A proximidade e o contato físico entre mãe e filhote são essenciais ao cuidado materno efetivo e à própria sobrevivência do filhote, assegurando a proteção e o conforto para um desenvolvimento físico, social e emocional saudável na infância. Mas, se por um lado o mecanismo de apego assegura a proximidade sobre a qual a relação mãe-filhote é construída, por outro, impõe a ambos um processo de desvinculação com o amadurecimento das competências do filhote e o desmame. Investigamos o desenvolvimento do vínculo de apego entre mães e filhotes de macacos-prego, bem como o grau de dependência do filhote em relação à mãe, usando dados sobre disposição espacial, responsabilidade pela manutenção do contato e conflito entre eles, do nascimento ao desmame dos filhotes.

Primeiramente, analisamos a evolução da relação espacial entre mãe e filhote, comparando a frequência mensal de três categorias de distância física entre eles: (1) em contato e até 1 metro; (2) entre 1,1 metro até 10 metros; (3) a mais de 10 metros. Em seguida, avaliamos o papel de mães e filhotes na manutenção da proximidade entre eles, com base nos comportamentos de "quebrar contato" (díade está em contato ou até 1 metro de distância e mãe ou filhote quebra contato ou se afasta) e "fazer contato" (díade está separada e mãe ou filhote se aproxima ou faz contato). Posteriormente, calculamos a frequência mensal com que cada membro da díade aproximou-se e afastou-se do outro. Para determinar qual membro da díade foi o principal responsável por manter a proximidade entre eles, utilizamos o Índice Proximidade (ou Índice de Hinde), dado pela seguinte fórmula: $n^{\circ}$ de aproximação filhotes

$\overline{\sum n^{o} \text { de aproximação filhotes }+\mathrm{n}^{0} \text { de aproximações mães }}$

Valores positivos indicam que o filhote é o maior responsável pelas iniciativas de aproximação e contato com a mãe, enquanto valores negativos indicam o padrão oposto (Hinde \& Atkinson, 1970). Além disso, analisamos o momento e o contexto do desmame alimentar e locomotor dos filhotes do nosso estudo e de conflitos entre mães e filhotes.

\section{Desenvolvimento do vínculo mãe-filhote em macacos-prego}

Entre o nascimento e o segundo mês de vida, todos os filhotes foram exclusivamente dependentes das mães para nutrição e transporte e permaneceram o tempo todo em contato com elas. Nesse período o Índice de Proximidade (IP) foi igual a zero, já que não houve quebra de contato entre eles. A partir do terceiro mês, os filhotes começaram a quebrar contato e a se distanciar de suas mães, dando início ao processo de locomoção e alimentação independente. Desse momento em diante, observamos um acelerado e constante decréscimo do tempo em que mães e filhotes permaneceram em contato e até um metro de distância, chegando a menos de $45 \%$ do tempo de observação no sexto mês de vida dos filhotes. A ausência de rejeições e conflitos mostra que o distanciamento se deu por iniciativa dos filhotes em explorar seu entorno, e não em decorrência de rejeições maternas. Na verdade, os valores negativos do IP mostram que as mães foram as principais responsáveis por manter a proximidade física com seus filhotes no momento em que eles começam a explorar o ambiente independentemente (Figura 2). 


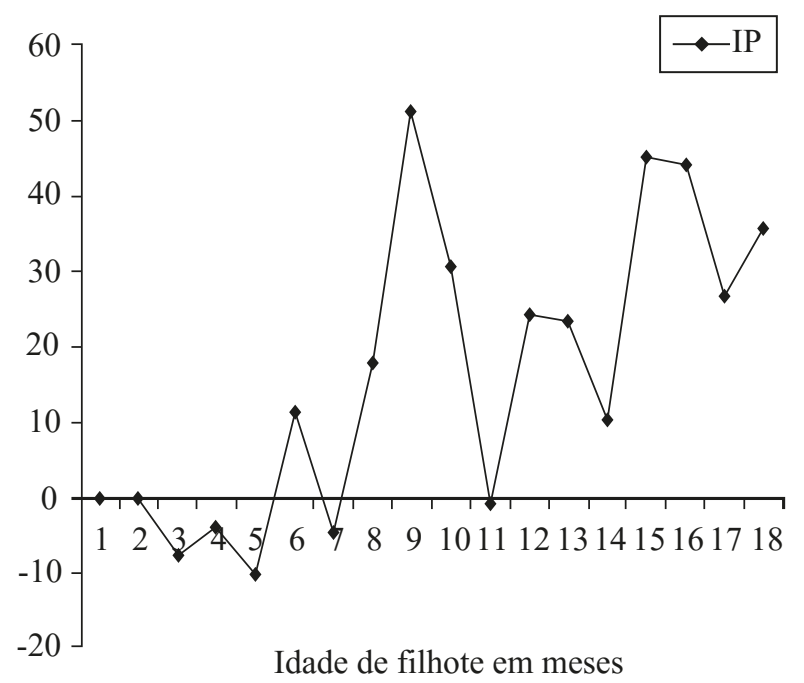

Figura 2. Distribuição dos valores do Índice de Proximidade (IP) entre mães e filhotes do primeiro ao $18^{\circ}$ mês de vida dos filhotes

No oitavo mês, o tempo de permanência em contato e até um metro entre mães e filhotes atingiu o patamar mais baixo ao longo de todo o primeiro ano de vida dos filhotes. Desse momento em diante, o IP se inverteu e passou a apresentar valores positivos, sinalizando que os filhotes assumiram a responsabilidade pela manutenção da proximidade com suas mães, padrão que se manteve até o final do $18^{\circ}$ mês (exceto pelo sétimo e $11^{\circ}$ mês Figura 2). $\mathrm{O}$ oitavo mês também foi marcado pelo início das rejeições maternas ao filhote e de conflitos resultantes dessas recusas, que indicam o início do processo de desmame. Chama a atenção que, exatamente nessa fase, os filhotes apresentaram mudanças comportamentais significativas que sugerem já estarem aptos a sobreviver por conta própria, dedicando tanto tempo quanto os adultos à locomoção e à alimentação independente. Em um caso, observamos um infante (Fábio) sobreviver após ser completamente desmamado por sua mãe (Física) no nono mês de vida (Verderane \& Izar, 2007), o que reforça nosso argumento de que no PET os filhotes de macacosprego são capazes de alimentar-se e locomover-se sozinhos antes do primeiro ano de vida.

Contudo, no nono mês observamos uma impressionante reação dos filhotes às recusas maternas em oferecer cuidado, com regressão dos comportamentos de alimentação e locomoção independente e correspondente aumento da amamentação e do transporte pela mãe, retornando ao padrão de desenvolvimento anterior ao oitavo mês. O maior pico de aproximações (IP) dos infantes em relação às suas mães nesse mês mostra que os filhotes foram os principais responsáveis por esse quadro (Figura 2).

A partir do primeiro ano, o contato foi sempre inferior a $25 \%$, mas mães e filhotes ainda permaneceram bastante tempo em um raio de dez metros de distância, cerca de $70 \%$ do tempo de observação ao final do $18^{\circ}$ mês de vida (Figura 3). Nota-se que, nessa fase, o processo de desmame acompanhado de resistência dos filhotes permanece, com dois ciclos de redução do tempo de contato e aumento da distância entre mães e filhotes, seguido de retornos a patamares anteriores (aumento do tempo em contato) associados a picos de aproximações dos filhotes, e subsequente redução do tempo de contato a valores ainda mais baixos.

Em média, o desmame alimentar ocorreu quando os filhotes tinham 16 meses de vida e o desmame locomotor quando tinham 13,5 meses. Assim, constatamos que a independência dos filhotes do nosso estudo, isto é, o momento que eles param por completo de mamar e ser transportados por suas mães, ocorreu cerca de um ano depois do início do processo de desmame, bem depois de já estarem aptos a se alimentar e locomover sozinhos.

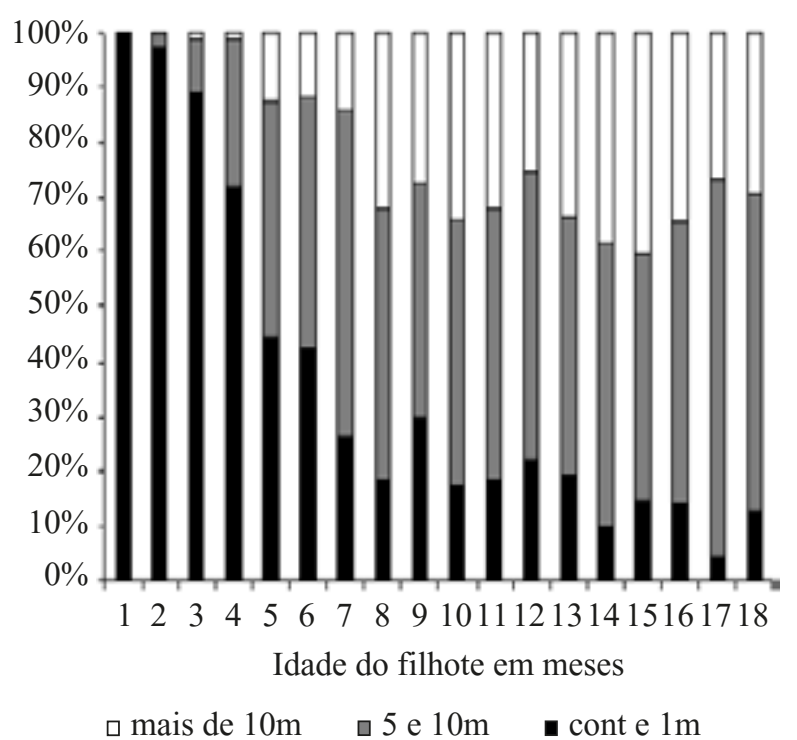

Figura 3. Distribuição das categorias de distância entre mães e filhotes do primeiro ao $18^{\circ}$ mês de vida dos filhotes

\section{Se os filhotes já podem sobreviver sozinhos, por que o desmame só ocorre mais tarde?}

Considerando que os filhotes foram os responsáveis pela manutenção do contato com sua mãe a partir do sexto mês de vida, sugerimos que eles tiveram um papel ativo no prolongamento do cuidado materno além de suas necessidades de sobrevivência. Portanto, o desmame tardio pode ser resultado da resistência dos filhotes em deixar de mamar e ser transportados. Mas a questão permanece: se os filhotes já são capazes de comer e locomover-se sozinhos, por que resistem em deixar de mamar e ser transportados?

Sugerimos que o período de dependência de filhotes primatas não está relacionado apenas à sua maturação física, mas também a processos psicológicos, especialmente o estabelecimento e desenvolvimento do vínculo com a mãe, e aprendizagem de habilidades complexas (Verderane, 2005). A imaturidade prolongada é típica dos primatas e está relacionada a 
um desenvolvimento mais lento, cérebros grandes e maior dependência de aprendizagem de habilidades típicas da espécie (Janson \& van Schaik, 1993; Joffe, 1997). Nesse aspecto, os macacos-prego destacam-se pela imaturidade dos filhotes ao nascimento e por um dos mais longos períodos de imaturidade dentre os primatas - cerca de $13 \%$ da vida entre o nascimento e a maturidade sexual, assumindo expectativa de vida de 40 anos (Fragaszy \& Bard, 1996) -, o que indica a importância do vínculo de apego do filhote com a mãe. Essa adaptação asseguraria as condições necessárias para o aprendizado do repertório comportamental complexo da espécie, que envolve técnicas sofisticadas de forrageamento, como o uso de ferramentas, e complexa estrutura social (Coelho et al., 2015; Fragaszy et al., 2013; Verderane et al., 2013; Resende, Ottoni, \& Fragaszy, 2008; Verderane, 2005). Esse padrão assemelha-se mais ao dos pongídeos e humanos do que de espécies de tamanho corpóreo similar ao de macacosprego (Fragaszy, Baer, \& Adams-Curtis, 1991).

Já o papel que a rejeição materna desempenha no processo de independência do filhote é mais controverso, com autores que a consideram um mecanismo que promove a independência do filhote (Schino, Speranza, $\&$ Troisi, 2001), e outros que argumentam o oposto, que ela aumenta o apego do filhote com a mãe (Bowlby, 1969). Nossos resultados apoiam parcialmente ambas as hipóteses. Num primeiro momento, as rejeições maternas provocaram um aumento da busca por cuidado materno nos filhotes, apesar de suas habilidades locomotoras e alimentares já estarem bem desenvolvidas nessa idade. Depois, parece haver uma acomodação ou "aceitação" dos filhotes e aos poucos a rejeição acaba promovendo a independência. Contudo, as mães não são imunes à resistência dos filhotes frente às suas recusas em fornecer cuidados, e reagem às intensas demandas afrouxando a rejeição e o processo de desmame, prolongando o vínculo de dependência.

\section{Por que as mães não rejeitam seus filhotes?}

De acordo com a teoria de investimento parental de Trivers (1972), o conflito entre mães e filhotes ocorre porque o cuidado parental aumenta as chances de sobrevivência do infante às custas da diminuição da habilidade reprodutiva dos pais. $\mathrm{O}$ conflito reside no fato de que para a mãe é mais vantajoso desvincular-se o quanto antes de seu filhote atual e investir na criação de outra prole, enquanto, para o filhote, o mais vantajoso é estender o período de cuidado pelo maior tempo possível.

Essa flexibilidade pode ser resultante de variabilidade de trajetórias ontogenéticas, afetadas por fatores ecológicos e sociais distintos. Portanto, é plausível supor que as bases comportamentais dessas diferenças se manifestem muito cedo no desenvolvimento do filhote e sejam moduladas por padrões específicos de vinculação mãe-filhote e estilos de cuidado resultantes de pressões sociais e ecológicas distintas.
Dessa forma, deveríamos esperar que as mães de macacos-prego resistissem às tentativas dos filhotes de manter os cuidados a partir do momento em que eles já são capazes de sobreviver sozinhos. Porém, o relacionamento da díade é um sistema social bipartido, no qual comportamento e fisiologia de mães e filhotes são mutuamente regulados. Assim, embora a maior parte dos estudos sobre o apego mãe-infante em primatas enfoque importância do apego para o desenvolvimento do filhote - revisão em Rosenblum e Paully (1991) -, é fato que os mecanismos comportamentais (ou psicológicos) asseguram também o vínculo parental da mãe em relação ao filhote (Maestripieri, 2001). Nosso estudo oferece uma contribuição adicional, mostrando que vínculo é um processo dinâmico em que mãe e filhote tornam-se mutuamente vinculados e desempenham papéis ativos, distintos e que se modificam ao longo da infância do filhote.

\section{Conclusão}

Neste estudo relatamos a existência de estilos de cuidado materno distintos em uma espécie de primata não humano do Novo Mundo, o macaco-prego. Vimos também que o apego é um processo dinâmico, em que mães e filhotes participam e respondem mutuamente ao comportamento do outro, e que esse vínculo entre eles foi mantido muito além das necessidades de sobrevivência dos filhotes. Apesar do número limitado de díades observadas, nossos achados são muito estimulantes e nos permitem propor algumas hipóteses que poderão ser testadas em investigações futuras.

É possível que a variabilidade de estilos de cuidado materno resultante da combinação de características de mães, filhotes e contextos socioecológicos, aliada ao prolongamento do vínculo de apego, pavimente caminhos para diferentes trajetórias de desenvolvimento. Esse pode ser um dos mecanismos pelos quais surgem e se consolidam as diferenças interindividuais nas populações adultas, assim como observado em populações humanas.

As incongruências entre os comportamentos maternos claramente associados à proteção e à rejeição (amamentação, transporte e proximidade com o filhote) e as demais variáveis de cuidado (catação e partilha de alimento) sugerem a existência de uma terceira dimensão do estilo materno, como recentemente tem-se sugerido na literatura. A chamada afetuosidade materna diz respeito à extensão de interações íntimas entre mãe e filhote (Maestripieri, Hoffman, Anderson, Carter, \& Higley, 2009), como o contato corporal íntimo e as interações face a face (Byrne \& Suomi, 2009), que guarda várias similaridades com as práticas parentais humanas, porém ainda é pouco compreendida em primatas não humanos.

Em um estudo que visou aprofundar a compreensão sobre a afetuosidade materna em macacos-prego selvagens, 
observamos que o repertório de interações íntimas entre mães e filhotes é mais extenso e sofisticado do que se supunha, e inclui várias formas de estimulação tátil do filhote e interações face a face (Verderane \& Izar, 2014). Investigar o papel desse tipo de interação para a qualidade e a extensão do vínculo de apego mãe-filhote e para o próprio desenvolvimento de habilidades socioemocionais dos filhotes em uma espécie filogeneticamente distante, em aspectos outrora considerados exclusivamente humanos, abre uma perspectiva comparativa fascinante para uma compreensão mais ampla das bases biológicas da parentalidade humana.

\section{Maternal care style in primates: considerations from a New World species}

Abstract: When aiming understand the human behavior, comparisons with nonhuman primates are especially relevant to identify homoplasies (similar characteristics that evolve independently in different species). In this paper, we present a two-year longitudinal study on the maternal behavior of capuchin monkeys (Sapajus spp.) under naturalistic conditions. Our results revel distinct maternal care styles within a continuum ranging from permissiveness (laissez-faire) to protectiveness. The observed development of mothers and infants bond suggests that the dependence period of capuchin monkeys infants involves, in addition to physical maturation processes, the establishment and development of psychological processes associated with the attachment system. It is possible that the variability of maternal styles - resulting from the combination of mothers' and infants' characteristics, as well as socioecological contexts, along with the extension of the attachment bond - are responsible for paving the way for different developmental trajectories. This may be one of the mechanisms underlying interindividual differences arise in adult populations, as seen in humans.

Keywords: attachment, mother-infant interaction, parenting care, infancy, Sapajus.

\section{Styles de soins maternels dans les primates : considerations d'une espèce du Nouveau Monde}

Résumé: Lorsque nous cherchons à comprendre le comportement humain, les comparaisons avec les primates non humains sont particulièrement pertinentes pour identifier les homoplasies (caractéristiques similaires qui évoluent indépendamment dans différentes espèces). Dans cet article, nous présentons les résultats d'une étude longitudinale de deux ans sur le comportement maternel du Sapajou capucin (Sapajus spp.) dans des conditions naturelles. Nos résultats nous ont permis d'identifier différents styles de soins maternels dans un continuum de permissivité à la protection. Nous avons observé que le modèle de lien d'attachement entre la mère et la progéniture suggère que la période de dépendance des bébés singes implique, en plus des processus de maturation physique, l'établissement et le développement de processus psychologiques associés au système d'attachement. II est possible que la variabilité des styles maternels résultant de la combinaison des caractéristiques de la mère, de la progéniture et du contexte socioécologique, ainsi que l'extension du lien d'attachement, ouvrent la voie à différentes trajectoires de développement. Comme les humains, il peut être un des mécanismes par lequel les différences interindividuelles apparaissent et se consolident au sein des populations adultes.

Mots-clés : Attachement, interaction mère-enfant, soins parentaux, enfance, Sapajus.

\section{Estilos de cuidado materno en primates: consideraciones a partir de una especie del Nuevo Mundo}

Resumen:Cuandobuscamosentenderelcomportamiento humano, comparacionescon primates no humanos son especialmente relevantes para identificar homoplasias (características similares que evolucionan independientemente en diferentes especies). En este artículo, presentamos los resultados de un estudio longitudinal de dos años sobre el comportamiento materno de monos capuchinos (Sapajus spp.) en condiciones naturales. Los resultados permitieron identificar estilos de cuidado materno distintos dentro de un continuo de permisividad a la protección. Se observó que el patrón de desarrollo del vínculo de apego entre madre y cría sugiere que el período de dependencia de la cría de monos capuchinos involucra, además de procesos de maduración física, el establecimiento y desarrollo de procesos psicológicos asociados al sistema de apego. Es posible que la variabilidad de estilos maternos resultante de la combinación de características de madres, crías y contextos socioecológicos, aliada a la prolongación del vínculo de apego, establezca caminos para diferentes trayectorias de desarrollo. Tal como en humanos, este puede ser uno de los mecanismos por los que surgen y se consolidan las diferencias interindividuales en las poblaciones adultas.

Palabras clave: apego, interacción madre-cría, cuidado parental, infancia, Sapajus. 


\section{Referências}

Ainsworth, M. S. (1979). Infant-mother attachment. American Psychologist, 34(10), 932-937. doi: 10.1037/0003066X.34.10.932

Ainsworth, M. S., \& Bowlby, J. (1991). An ethological approach to personality development. American Psychologist, 46(4), 333-341. doi: 10.1037/0003-066X.46.4.333

Altmann, J. (1974). Observational study of behavior sampling methods. Behaviour, 49, 227-267. doi: 10.1111/j.17487692.1999.tb00784.x

Altmann, J. (1980). Baboons mothers and infants. Cambridge, MA: Harvard University Press.

Badinter, E. (1985). Um amor conquistado: o mito do amor materno. Rio de Janeiro, RJ: Nova Fronteira.

Bercovitch, F. B. (2002). Sex-biased parental investment in primates. International Journal of Primatology, 23(4), 905-921. doi: 10.1023/A:1015585117114

Berman, C. M. (1980). Mother-infant relationships among free-ranging rhesus monkeys on Cayo Santiago: a comparison with captive pairs. Animal Behaviour, 28(3), 860-873. doi: 10.1016/S0003-3472(80)80146-1

Berman, C. M., Rasmussen, K. L. R., \& Stephen, J. S. (1997). Group size, infant development and social networks in free-ranging rhesus monkeys. Animal Behaviour, 53, 405-421. doi: 10.1006/anbe.1996.0321

Bowlby, J. (1969). Apego: a natureza do vínculo. São Paulo, SP: Martins Fontes.

Byrne, R. (1995). The thinking ape: evolutionary origins of intelligence. New York, NY: Oxford University Press.

Byrne, G., \& Suomi, J. (2009). Intimate social behavior in infant interactions in Cebus apella. American Journal of Primatology, 71, 77-85. doi: 10.1002/ajp.20626

Carminatti, M. O. F. (2009). Efeito da paridade da mãe $e$ do sexo do filhote sobre o estilo de cuidado materno $e$ desenvolvimento de filhotes de macacos-prego (Cebus sp) (Dissertação de Mestrado). Instituto de Psicologia, Universidade de São Paulo, São Paulo.

Carvalho, R. V. C., Seidl-de-Moura, M. L., Martins, G. D. F., \& Vieira, M. L. (2014). Culture and developmental trajectories: a discussion on contemporary theoretical models. Early Child Development and Care, 184(11), 1599-1614. doi: 10.1080/03004430.2013.871273

Chatterjee, H. J., Ho, S. Y. W., Barnes, I., \& Groves, C. (2009). Estimating the phylogeny and divergence times of primates using a supermatrix approach. BMC Evolutionary Biology, 9, 1-19. doi: 10.1186/1471-2148-9-259

Coelho, C. G., Falótico, T., Izar, P., Mannu, M., Resende, B. D., Siqueira, J. O., \& Ottoni, E. B. (2015). Social learning strategies for nut-cracking by tufted capuchin monkeys (Sapajus spp.). Animal Cognition, 18(4), 911919. doi: 10.1007/s10071-015-0861-5

Dettmer, A. M., Kaburu, S. S. K., Byers, K. L., Murphy, A. M., Soneson, E., Wooddell, L. J., \& Suomi, S. J. (2016). First-time rhesus monkey mothers, and mothers of sons, preferentially engage in face-to-face interactions with their infants. American Journal of Primatology, 78(2), 238-246. doi: 10.1002/ajp.22503

Fairbanks, L. A. (2003). Parenting. In D. Maestripieri (Ed.). Primate psychology (pp. 144-170). Cambridge, MA: Harvard University Press.

Falótico, T., Verderane, M. P., Resende, B., Ottoni, E. B., \& Izar, P. (2003). Rank reversal in females hierarchy in semi-free ranging capuchin monkeys (Cebus apella). In Resumos do $28^{\circ}$ International Ethological Conference. Florianópolis, SC. doi: 14293/S2199-1006.1.SOR-.PPP7BBF.v1

Fragaszy, D. M., Baer, J., \& Adams-Curtis, L. (1991). Behavioral development and maternal care in tufted capuchins (Cebus apella) and squirrel monkeys (Saimiri sciureus) from birth through seven months. Developmental Psychobiology, 24(6), 375-393. doi: $10.1002 /$ dev.420240602

Fragaszy, D. M., \& Bard, K. (1996). Comparison of development and life history in Pan and Cebus. International Journal of Primatology, 18(5), 683-701. doi: 10.1023/A:1026339712071

Fragaszy, D. M., Biro, D., Eshchar, Y., Humle, Y., Izar, P., Resende, B. D., \& Visalberghi, E. (2013). The fourth dimension of tool use: temporally enduring artefacts aid primates learning to use tools. Philosophical Transactions of the Royal Society of London B: Biological Sciences, 368, 1-10. doi: 10.1098/rstb.2012.0410

Fragaszy, D. M., Visalberghi, E., \& Fedigan, L. (2004). The complete capuchin. Cambridge, MA: Cambridge University Press.

Hinde, R. A., \& Atkinson, S. (1970). Assessing the role of social partners in maintaining mutual proximity, as exemplified by mother-infant relations in rhesus monkeys. Animal Behaviour, 18, 169-176. doi: 10.1016/0003-3472(70)90087-4

Hrdy, S. B. (1999): Mother nature: a history of mothers, infants and natural selection. New York, NY: Pantheon.

Izar, P., \& Ferreira, R. G. (2007). Socioecologia de macacosprego (Cebus apella) selvagens e provisionados: uma análise comparativa. In J.C. Bicca-Marques (Ed.). A primatologia no Brasil 10 (pp. 323-338). Porto Alegre, RS: Sociedade Brasileira de Primatologia.

Izar, P., Verderane, M. P., Peternelli, L. S., MendonçaFurtado, O., Presotto, A., Tokuda M., Visalberghi, E., \& Fragaszy, D. M. (2012). Flexible and conservative features of social systems in tufted capuchin monkeys: comparing the socioecology of Sapajus libidinosus and Sapajus nigritus. American Journal of Primatology, 74, 315-331. doi: 10.1002/ajp.20968

Izar, P., Verderane, M. P., Visalberghi, E., Ottoni, E. B., Oliveira, M. G., Shirley, J., \& Fragaszy, D. M. (2006). Cross-genus adoption of a marmoset (Callithrix jacchus) by wild capuchin monkeys (Cebus libidinosus): case report. American Journal of Primatology, 68, 692-700. doi: 10.1002/ajp.20259 
Janson, C. H., \& van Schaik, C. P. (1993). Ecological risk aversion in juvenile primates: slow and steady wins the race. In M. Pereira \& L. Fairbanks (Eds.). Juvenile primates (pp. 62-73). New York: Oxford University Press.

Joffe, T. H. (1997). Social pressures have selected for an extended juvenile period in primates. Journal of Human Evolution, 32, 593-605. doi: 10.1006/jhev.1997.0140

Li, T., Ren, B., Li, D., Zhu, P., \& Li. M. (2013). Mothering style and infant behavioral development in yunnan snub-nosed monkeys (Rhinopithecus bieti) in China. International Journal of Primatology, 34, 681-695. doi: 10.1007/s10764-013-9687-7

Lonsdorf, E. V. (2017). Sex differences in nonhuman primate behavioral development. Journal of Neuroscience Research, 95(1-2), 213-221. doi: 10.1002/jnr.23862

Maestripieri, D. (1999). The biology of human parenting: insights from nonhuman primates. Neuroscience and Biobehavioral Reviews, 23, 411-422. doi: 10.1016/ S0149-7634(98)00042-6

Maestripieri, D. (2001). Intraspecific variability in parenting styles of rhesus macaques (Macaca mulatta): the role of the social environment. Ethology, 107, 237-248. doi: 10.1046/j.1439-0310.2001.00661.x

Maestripieri, D., Hoffman, C. L., Anderson, G. M., Carter, S., \& Higley, J. D. (2009). Mother-infant interactions in free-ranging rhesus macaques: relationships between physiological and behavioral variables. Physiology \& Behavior, 96, 613-619. doi: 10.1016/j. physbeh.2008.12.016

Maestripieri, D., Ross, S. K., \& Megna, N. L. (2002). Motherinfant interactions in western lowland gorillas (Gorilla gorilla): spatial relationships, communication and opportunities for social learning. Journal of Comparative Psychology, 116(3), 219-227. doi:10.1037/07357036.116.3.219

Okamoto-Barth, S., Tanaka, M., Kawai, N., \& Tomonaga, M. (2007). Looking compensates for the distance between mother and infant chimpanzee. Developmental Science, 10(2), 172-182. doi: 10.1111/j.1467-7687.2007.00547.x

Pereira, M. E., \& Altmann, J. (1985). Development of social behavior in free living nonhuman primates. In Watts, E. S. (Ed.). Nonhuman primate models for human growth and development (pp. 217-309). New York: Alan R. Liss.

Resende, B. D. (2004). Ontogenia de comportamentos manipulativos em um grupo de macacos-prego (Cebus apella) em situação de semiliberdade (Tese de Doutorado). Instituto de Psicologia, Universidade de São Paulo, São Paulo.

Resende, B. D, Ottoni, E. O., \& Fragaszy, D. M. (2008). Ontogeny of manipulative behavior and nut-cracking in young tufted capuchin monkeys (Cebus apella): a perception-action perspective. Developmental Science, 11(6), 828-840. doi: 10.1111/j.1467-7687.2008.00731.x

Rosenblum, L. A., \& Andrews, M. W. (1994). Influences of environmental demand on maternal behavior and infant development. Acta Paediatrica, 85, 57-63. doi: 10.1111/ j.1651-2227.1994.tb13266.x

Saltzman, W., \& Maestripieri, D. (2011). The neuroendocrinology of primatematernal behavior.Progress in Neuro-Psychopharmacology \& Biological Psychiatry, 35, 1192-1204. doi: 10.1016/j.pnpbp.2010.09.017

Schino, G., Speranza, L., \& Troisi, A. (2001). Early maternal rejection and later social anxiety in juvenile and adult Japanese macaques. Developmental Psychobiology, 38, 186-190. doi: 10.1002/dev.1012

Tinbergen, N. (1963). On aims and methods of ethology. Zeitschrift für Tierpsychologie, 20(4), 410-433.

Trivers, R. L. (1972). Parental investment and sexual selection. In B. Campbell (Ed.). Sexual selection and the descent of man (pp. 136-179). London: Heinemann.

Trivers, R. L. (1974). Parent-offspring conflict. Integrative and Comparative Biology, 14(1), 249-264. doi: 10.1093/ $\mathrm{icb} / 14.1 .249$

Verderane, M. P. (2005). Estilos de cuidado materno e desenvolvimento das relações sociais de infantes de macacos-prego, Cebus apella, de 0 a 18 meses de idade. (Dissertação de Mestrado). Instituto de Psicologia, Universidade de São Paulo, São Paulo.

Verderane, M. P., \& Izar, P. (2007). O cuidado alomaterno exibido por uma fêmea de macaco-prego (Cebus apella) após a morte da própria cria: um caso de adoção? In J. C. Bicca-Marques. A primatologia no Brasil (Vol. 10, pp. 463-476). Porto Alegre, RS: Sociedade Brasileira de Primatologia.

Verderane, M. P., \& Izar, P. (2014). Early intimate interactions between mother and infant in wild bearded capuchin monkeys (Sapajus libidinosus). In Abstract of $25^{\circ}$ Congress of Primatological Society (p. 789). Hanoi, Vietnam.

Verderane, M. P., Izar, P., Visalberghi, E., \& Fragaszy, D. M. (2013). Socioecology of wild bearded capuchin monkeys (Sapajus libidinosus): an analysis of social relationships among female primates that use tools in feeding. Behaviour, 150, 659-689. doi: 10.1163/1568539X-00003076

Yamamoto, M. E., Araujo, A., Arruda, M. F., Lima, A. K. M., Siqueira, S. O., \& Hattori, W. T. (2014). Male and female breeding strategies in a cooperative primate. Behavioural Processes, 109, 27-33. doi: 10.1016/j.bepr

Recebido: 31/07/2019 Aprovado: 04/08/2019 